\title{
Novo sistema de cardioplegia sangüínea em cirurgia de cardiopatia congênita
}

\author{
Pedro R. SALERnO *, Magaly Santos ARRAES *, Marcelo B. JATENE *, Fábio B. JANTENE *, \\ Paulo CHACCUR ${ }^{* *}$, Jarbas J. DINKHUYSEN ${ }^{* *}$, Camilo ABDULMASSIH NETO ${ }^{* *}$, Antoninho S. \\ ARNONI $^{* * *}$, Paulo P. PAULISTA ${ }^{* * *}$, Adib D. JATENE ${ }^{\star}$, Luiz Carlos Bento de SOUZA*
}

SALERNO, P. R.; ARRAES, M. S.; JATENE, M. B.; JATENE, F. B.; CHACCUR, P.; DINKHUYSEN, J. J.; ABDULMASSIH NETO, C.; ARNONI, A. S.; PAULISTA, P. P.; JATENE, A. D.; SOUZA, L. C. B. - Novo sistema de cardioplegia sangūinea em cirurgia de cardiopatia congênita. Rev. Bras. Cir. Cardiovasc., 10 (3): 150-153, 1995

RESUMO: O objetivo do presente estudo é apresentar um sistema de cardioplegia sangũinea para cirurgla de cardiopatia congênita. Foram analisados, prospectivamente, 71 pacientes com $10.12 \mathrm{~kg}$ em média, 34 eram do sexo feminino e a idade média foi de 2,1 anos. Da linha arterial, passando por um trocador de calor, aspiramos para uma seringa de $50 \mathrm{cc}$ sangue a $8^{\circ} \mathrm{c}$, a qual é conectada a outra seringa de $10 \mathrm{cc}$ com uma soluçāo decimal de potássio, através de duas torneirinhas. A mistura da soluçāo decimal ( $3 \mathrm{ml}$ ) com $47 \mathrm{ml}$ de sangue a $8^{\circ} \mathrm{C}$ origina uma solução de sangue com $15 \mathrm{meq} / 1$ de potássio. Esta soluçăo é infundida na raiz da aorta, de acordo com o peso do paciente $(10 \mathrm{cc} / \mathrm{kg})$. Em todos obtivemos parada cardioplégica. O tempo médio de extracorpórea fol de 87,2 minutos e de pinçamento aórtico, de 60,7 minutos. Treze pacientes evoluíram para óbito, 6 por falência miocárdica, 3 por sindrome de baixo débito, 2 com arritmia, 1 com falência renal $\theta$ 1 com coagulopatia. Os 58 demais pacientes receberam alta hospitalar sem complicaçöes. Em conclusăo, este método mostrou ser eficiente na preservaçăo miocárdica e com baixa morbi-mortalidade.

DESCRITORES: Cardioplegia. Cardioplegia, cirurgia de cardiopatias congênitas. Proteção miocárdica, cardioplegia. Miocárdio, proteçăo, cardioplegia.

\section{INTRODUÇĀO}

Os recentes avanços nos métodos diagnósticos e a melhoria na técnica operatória resultaram em um aumento no número de crianças e neonatos que são submetidos a procedimentos cirúrgicos, que requerem o uso de circulação extracorpórea e de algum método de proteção miocárdica.

Existem diferenças importantes em termos estruturais 5,20 , de metabolismo $3,14,17$ e de função entre o miocárdio de neonato $e$ do adulto $3,11,14,24$.
A preservaçāo miocárdica para esse crescente seguimento de pacientes é um elemento crucial para reduzir a morbi-mortalidade. Com este intuito, vários tipos de soluçőes cardioplégicas e de sistemas de cardioplegia foram desenvolvidos $1,7,8,12,16,18,21$.

O objetivo deste trabalho é apresentar um sistema simples de entrega de cardioplegia sangüinea, desenvolvido experimentalmente e aplicado clinicamente em pacientes submetidos à correçăo cirúrgica de cardiopatias congênitas.

\footnotetext{
Trabalho realizado no Hospital do Coraçāo da Associaçăo do Sanatorio Sirio e no Instituto Dante Pazzanese de Cardiologia. Săo Paulo, SP, Brasil Apresentado ao $22^{\circ}$ Congresso Nacional de Cirurgia Cardiaca. Brasilia, DF, 30 de março a $1^{2}$ do abril, 1995.

* Do Hospital do Coraçăo da Associaçăo do Sanatório Sírio.

** Do Hospital do Coraçāo e do Instituto Dante Pazzanese de Cardiologia.

*** Do Instituto Dante Pazzanese de Cardiologia.

Endereço para correspondéncia: Pedro Salerno. Hospital do Coração. Rua Desembargador Elizeu Guilherme, 123. CEP: 04004-030 São Paulo, SP, Brasil. Tel: (011) 887.6611 - Fax: (011) 889.8698
} 
SALERNO, P. R.; ARRAES, M. S.; JATENE, M. B.; JATENE, F. B.; CHACCUR, P.; DINKHUYSEN, J. J.; ABDULMASSIH NETO, C.; ARNONI, A.S.; PAULISTA, P. P.; JATENE, A. D.; SOUZA, L. C. B. - Novo sistema de cardioplegia sangũinea em cirurgia de cardiopatia congenita, Rev. Bras. Cir. Cardiovasc., 10 (3): 150-153, 1995.

\section{MATERIAL E MÉTODOS}

No periodo de dezembro de 1993 a janeiro de 1995 , foram analisados prospectivamente 71 pacientes com idade média de 2,1 anos (2 dias - 12 anos), 37 do sexo masculino e 34 do sexo feminino, com peso médio de $10,12 \mathrm{~kg}(2390 \mathrm{~kg}-31.0 \mathrm{~kg})$.

Estes pacientes eram portadores das seguintes lesōes: comunicação interventricular 13 pacientes (pts), defeito do septo atrioventricular 11 pts, tetralogia de Fallot 11 pts, drenagem anômala das veias pulmonares $7 \mathrm{pts}$, transposiçāo das grandes artérias 7 pts, atresia pulmonar 4 pts, dupla via de saida de ventrículo direito 3 pts, comunicação interatrial $3 \mathrm{pts}$, atresia tricúspide $2 \mathrm{pts}$, ventrículo único 2 pts, estenose subaórtica 2 pts, estenose valvar aórtica $1 \mathrm{pt}$, interrupção do arco aórtico $1 \mathrm{pt}$, origem anómala de coronária esquerda $1 \mathrm{pt}$, estenose pulmonar pós operação de Jatene 1 pt, insuficiência mitral 1 pt e hipoplasia do coraçăo esquerdo 1 paciente.

O sistema consiste de um mini-trocador de calor conectado à linha arterial através de um tubo; o sangue passa pelo trocador e vai ao campo operatório através de outro tubo. Na extremidade deste há duas torneirinhas conectadas a duas seringas de $50 \mathrm{cc}$ e $10 \mathrm{cc}$. Na seringa de $50 \mathrm{cc}$ é aspirado sangue arterializado a $8 \mathrm{C}$; na seringa de $10 \mathrm{cc}$ é preparada soluçăo decimal de $\mathrm{KCL}$ a $19,1 \%$. Manuseando as seringas, injetamos $3 \mathrm{ml}$ de solução de $\mathrm{KCL}$ na seringa de $50 \mathrm{cc}$ e aspiramos $47 \mathrm{ml}$ de sangue da linha arterial, originando uma mistura de sangue com concentração de potássio de $15 \mathrm{meq} /$ I. Esta solução é injetada na raiz da aorta. A dose de cardioplegia varia de acordo com o peso da criança $(10 \mathrm{cc} / \mathrm{kg})$. A cada 30 minutos, outra dose é infundida com $2 \mathrm{ml}$ de soluçâo de potássio (10 $\mathrm{meq} / 1$ ) para $48 \mathrm{ml}$ de sangue (Figura 1).

\section{RESULTADOS}

Obtivemos parada da atividade elétrica do coração em todos os pacientes. O tempo médio de circulaçăo extracorpórea foi de $87,2 \mathrm{~min}$ (30-230 $\mathrm{min}$ ), e pinçamento aórtico médio de $60,7 \mathrm{~min}(20-163)$.

$\mathrm{Na}$ saida de circulaçăo extracorpórea, a pressão arterial média foi de $53,4 \mathrm{mmHg}(40-75 \mathrm{mmHg})$, pressão de átrio esquerdo médio de $8,8 \mathrm{mmHg}(5$ $14 \mathrm{mmHg}$ ) e recebendo em média $4,7 \mathrm{mg} / \mathrm{kg} / \mathrm{min}$ de dopamina nas primeiras 8 horas de pós-operatório.

Ocorreram 13 óbitos hospitalares, 6 por disfunção pulmonar, 3 por sindrome de baixo débito, 2 com arritmia, 1 com insuficiência renal aguda $\theta 1$ por coagulopatia. Os demais pacientes receberam alta hospitalar em boas condiçōes clínicas.

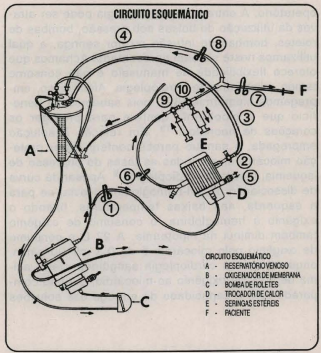

Fig. 1

\section{COMENTÁRIOS}

A má proteção miocárdica durante a correção de cardiopatias congênitas é responsável por uma porcentagem elevada de óbitos no pós-operatório imediato ${ }^{5}$. A optimizaçăo da preservação miocárdica permite obter melhores resultados, principalmente nos dias de hoje, onde novos procedimentos foram desenvolvidos para corrigir cardiopatias congênitas complexas $6,10,17$.

A utilizaçăo do circuito de extracorpórea para estes procedimentos torna-se complicada por dificuldades técnicas associadas ao tamanho do paciente e devido à multiplicidade e complexidade dos defeitos cardiacos. O que, para um paciente adulto, são procedimentos rotineiros, em termos de condução da circulação extracorpórea e proteçăo miocárdica, nos pacientes com cardiopatia congênita e baixo peso torna-se mais complicado.

A proteção miocárdica para estes pacientes é um elemento crucial para reduzir a morbi-mortalidade. A estratégia empregada para realizar a preservação miocárdica em neonatos e crianças com cardiopatia congênita tem que ser adaptada para uma grande variedade de peso e tamanho dos pacientes, técnicas de entrega de cardioplegia, soluçōes cardioplégicas, instituiçōes e cirurgiôes. Devemos utilizar algo simples, seguro, de baixo custo e que não ocupe muito espaço e tempo no campo 
SALERNO, P. R.; ARRAES, M. S.; JATENE, M. B.; JATENE, F. B.; CHACCUR, P.; DINKHUYSEN, J. J.; ABDULMASSIH NETO, C.; ARNONI, A. S.; PAULISTA, P. P.; JATENE, A. D.; SOUZA, L. C. B. - Novo sistema de cardioplegia sangüinea em cirurgia de cardiopatia congênita. Rev. Bras. Cir. Cardiovasc., 10 (3): 150-153, 1995.

operatório. A entrega de cardioplegia pode ser através da utilizaçāo de bolsas sob pressăo, bombas de roletes, bombas de infusão ou por seringa, a qual utilizamos neste sistema apresentado. Achamos que oferece flexibilidade de manuseio e não consome tempo administrando cardioplegia. Além disso, empregamos hipotermia $(8 C)$, pois sabemos do benefício que a hipotermia confere para proteger os coraçōes de neonatos 13 . Em relaçăo à solução empregada, o sangue parece conferir maior proteção miocárdica, em todas as fases do processo de isquemia, à parada cardioplégica ${ }^{9}$. Apesar da curva de dissociação da oxi-hemoglobina desviar-se para a esquerda, nas baixas temperaturas, fixando o oxigênio à hemoglobina, o consumo de oxigênio também diminui na hipotermia. A 22 C o consumo de oxigênio pelo miocárdio é de $0,3 \mathrm{ml} / 100 \mathrm{~g} / \mathrm{min}$, enquanto que a cardioplegia sangũinea oferta acima de $50 \%$ de oxigênio ao miocárdio, quando comparado com a capacidade de entrega das soluçōes cristalóides 2, 4. O sangue também possui uma capacidade tampäo importante, o que, para os corações de neonatos, é significativo, pois estes coraçōes têm uma tendência maior à acidose, devido à utilizaçāo da via glicolítica com maior produçăo de lactato ${ }^{20}$. Outro benefício, ainda em estudos, é o do sangue ter capacidade de previnir as lesōes de reperfusăo ${ }^{20}$. Apesar de vários trabalhos clínicos atribuírem bons resultados utilizando a cardioplegia cristalóide, os dados experimentais e clínicos, nestes últimos anos, têm conferido à cardioplegia sangüinea como o veículo de escolha, principalmente nos coraçōes que vêm para cirurgia com algum tipo de injúria 23 .

Com estes dados e resultados, podemos considerar que este sistema apresentado preenche os requisitos fisiológicos para preservação miocárdica, sem onerar e complicar a utilizaçăo da cardioplegia.

SALERNO, P. R.; ARRAES, M. S.; JATENE, M. B.; JATENE, F. B; CHACCUR, P ; DINKHUYSEN, J. J.; ABDULMASSIH NETO, C.; ARNONI, A. S.; PAULISTA, P. P.; JATENE, A. D.; SOUZA, L. C. B. - New delivery system for pediatric blood cardioplegia. Rev. Bras. Cir. Cardiovasc., 10 (3): 150-153 , 1995.

ABSTRACT: The purpose of this study is to present a simple Delivery System of Blood Cardioplegia for myocardial preservation during congenital cardiac operation. We prospectively analysed 71 patients (pts) with $10 / 12 \mathrm{~kg}$ of body mean weight, 34 were female and mean age was $2 / 1$ years. From the arterial line, passing through a mini heat exchanger, blood at $8 \mathrm{C}$ is aspirated to a $50 \mathrm{cc}$ syringe, which is conected to a $10 \mathrm{cc}$ syringe with a $\mathrm{KCL}+$ decimal solucion using 2 stopcocks. The mixture originates a cold blood with $15 \mathrm{mEg} / \mathrm{lof} \mathrm{KCL}$ + that is infused into the aortic root, according to the pts weight $(10 \mathrm{cc} / \mathrm{kg})$. In all pts cardiac arrest was obtained The mean extracorporeal circulation time and crossclamping was 87.2 and $60.7 \mathrm{~min}$. All pts recovered sinus rhythm with good ventricular function. Thirteen patients died, 6 with respiratory fallure, 3 with low cardiac output, 2 with dysrhythmias, 1 with renal failure and 1 with hematologic disorder; the other 58 patients left the hospital with no complications. In conclusion, this method showed good results with efficient myocarcial preservation and low morbi-mortality.

DESCRIPTORS: Cardioplegia. Cardioplegia, surgery for congenital cardiopathies. Myocardial protection, cardioplegia.

\section{REFERÊNCIAS BIBLIOGRÁFICAS}

1 AOKI, M.; NOMURA, F.; MAYER, J. E. - Interactions between preischemic hypothermia and cardioplegic solutions in the neonatal lamb heart. J. Thoracic. Cardiovasc. Surg., 107: 822-828, 1994.

BARNER, H. B. - Blood cardioplegia: a review and comparison with crystalloid cardioplegia. Ann. Thoracic. Surg., 52: 1354-1367, 1991.
3 BERS, D. M.; PHILIPSON, K. D.: LANGER, G. A. Cardiac contractility and sarcolemmal calcium binding in several cardiac muscle preparations. Am. $J$. Physiol, 240: H576-H583, 1981.

4 BUCKBERG, G. D.; BRAZIER, J. R.; NELSON, R. L. Studies of the effects of hypothermia on regional myocardial blood flow and metabolism during cardiopulmonary bypass. J. Thoracic. Cardiovasc. Surg., 73: 87-94, 1977. 
SALERNO, P. R.; ARRAES, M. S.; JATENE, M. B.; JATENE, F. B.; CHACCUR, P.; DINKHUYSEN, J. J.; ABDULMASSIH NETO C.; ARNONI, A. S.; PAULISTA, P. P.; JATENE, A. D.; SOUZA, L. C. B. - Novo sistema de cardioplegia sangúinea em cirurgia de cardiopatia congênita. Rev. Bras. Cir. Cardiovasc., 10 (3): 150-153, 1995.

BULL, C.; COOPER, J.; STARK, J. - Cardioplegic protection of the child's heart. J. Thorac. Cardiovasc. Surg., 88: 287-293, 1984.

CASTANEDA, A. R.; MAYER Jr., J. E.; JONAS, R. A.; LOCK, J. E.; WESSEL, D. L.; HICHEY, P. - The neonate with critical congenital heart disease: a surgical challenge. J. Thorac. Cardiovasc. Surg., 98: 869-875, 1989.

7 DENNY, T. L.; HAGGERTY, S. P.; BOLEY, T. M. CURTIS, J. J. - Lack of cardioplegia uniformity in clinical myocardial preservation. Ann. Thorac. Surg., 57: 648-651, 1994.

8 DRINKWATER, D. C.\& LAKS, H. - Pediatric cardioplegic techniques. Semin. Thorac. Cardiovasc. Surg., 5: 168-175, 1993.

9 DRINKWATER, D. C.; CUSHEN, C. K.; LAKS, H.: BUCKBERG, G. D. - The use of combined antegraderetrograde infusion of blood cardioplegic solution in pediatric patients undergoing heart operations. $J$. Thorac. Cardiovasc. Surg., 104: 1349-1355, 1992.

10 ESPOSITO, G.; KEETON, B. B.; SUTHERLAND, G. R.; MONRO, J. L.; MANNERS, J. M. - Open heart surgery in the first 24 hours of life. Ped. Cardiol, 10: 33-36, 1989 .

11 GEORGE, B. L.; NAKANISSHI, T.; NISHIOKA, K.; JARMAKANI. J. M. - Effect of verapamil on mechanical function of the neonatal rabbit heart. Ped. Res., 15: 463, 1981 (Resumo).

12 HEINLE, J. S; LODGE, A. J.; MAULT, J. R.; WHITAKER E. G.; UNGERLEIDER, R. M. - Myocardial function is normal after rapid cooling of the in vivo neonatal heart. Ann. Thorac. Surg., 57: 326-333, 1994.

13 HOSSEINZADEH, T.; TCHERVENKOV, C.; QUANTZ, M.; CHIU R. C. J. - Adverse effect of prearrest hypothermia in immature hearts: rate versus duration of cooling. Ann. Thorac. Surg., 53: 464-471, 1992.

JARMAKANI, J. M.; NAGATOMO, T.; LANGER, G. A. - The effect of calcium and high energy phosphate compounds on myocardial contracture in the newborn and adult rabbit. J. Mol. Cell. Cardiol, 10: 1017 . 1023,1978

15 JULIA, P. L.; BUCKBERG, G. D.; ACAR, C. - Studies of controlled reperfusion after ischemia, $X X \mid$ Reperfusate Composition: superiority of blood cardioplegia over crystalloid cardioplegia in limiting reperfusion damage importance of endogenous oxygen free radical scavengers in red blood cells. $J$. Thorac. Cardlovasc. Surg., 101: 303-313, 1991.

16 KOHMAN, L. J. \& VEIT, L. J. - Single-dose versus multidoses cardioplegia in neonatal hearts. J. Thorac. Cardiovasc. Surg., 107: 1512-1518, 1994.

17 LAKS, H. - Advances in the repair of complex congenital heart disease. Ped. Ann. 11: 926-931, 1982.

18 NISHIOTA, K.; NAKANISHI, T.; GEOORGE, B. L.; JARMAKANI, J.M. - The effect of calcium on the inotrophy of catecholamine and paired electrical stimulation in the newborn and adult myocardium. J. Mol. Cell Cardiol., 13: 511-520, 1981.

19 PEARL, J. M.: LAKS, H.; DRINKWATER, D. C.: MENESHIAN, A.; SUN, B.; GATES, R. N.; CHANG, P. - Normocalcemic blood or crystalloid cardioplegia provides better protection than does low-calcium cardioplegia. J. Cardiovasc. Surg., 105: 201-206, 1993.

20 REEVES, R. B. \& MALAN, A. - Model studies of intracellular acid-base temperature responses in ectotherms. Respir. Physiol., 28: 49-57, 1970.

21 ROSS, B. A.; SEIDEL, C. L.; GARSON, A.; MCNAMARA, D. - Verapamil effect on vascular smoth muscle: variation in immature and mature animals. Ped. Cardiol., 5: 252, 1984. (Resumo).

SHUN - TIM, D.; TCHERVENKOV, C. I.: LOUGH, J. O.: CHIU, R. C. J. - Newborn myocardial protection after prolonged prearrest cooling: a calcium overlood phenomenon? Ann. Thorac. Surg., 57: 311-318, 1994.

VINTEN - JOHANSEN, J.; EDGERTON, T. A.; HANSEN, K. J. - Surgical revascularization of acute (1 hour) coronary occlusion: blood versus crystalloid cardioplegia. Ann. Thorac. Surg., 42: 247-254, 1986.

YEE, E. S. \& EBERT, P. A. - Effect of ischemia on ventricular function, compliance and edema in immature and adult canine hearts. Surg. Forum, 30: 250-252, 1979 